\title{
EXPLORATORY SEARCH METHODOLOGY FOR SENTINEL 2 DATA: A PROSPECT OF BOTH VISUAL AND LATENT CHARACTERISTICS.
}

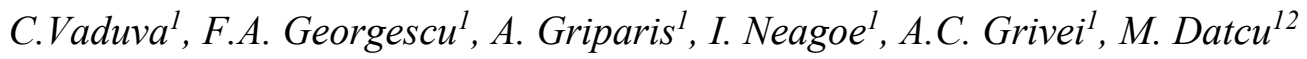 \\ ${ }^{1}$ Research Centre for Spatial Information, University Politehnica of Bucharest (UPB), Romania \\ ${ }^{2}$ Remote Sensing Technology Institute, German Aerospace Center (DLR), Germany
}

\begin{abstract}
Sentinel 2 (S2) satellite provides a systematic global coverage of land surfaces, measuring physical properties within 13 spectral intervals at a temporal resolution of 5 days. Computer-based data analysis is highly required to extract similarity by processing and assist human understanding and semantic annotation in support of Earth surface mapping. This paper proposes an exploratory search methodology for S2 data underpinning both visual and latent characteristics by means of data visualization and content representation. For optimized results, the authors focus on a detailed assessment of top relevant state-of-the-art algorithms for features extraction and classification to determine which one could handle best the characteristics of S2 data.
\end{abstract}

Index Terms - exploratory multispectral data analysis

\section{INTRODUCTION}

The need to understand the environment and to monitor the surrounding processes and activities has encouraged the progress of Earth Observation (EO) field, including remote sensing technology. Imaging sensors have been designed and developed such that they record specific aspects of Earth land cover based on spatial, spectral or radiometric resolution which can help estimate geophysical parameters and land processes. Part of the Copernicus Program, the Sentinel 2 (S2) mission targets terrestrial observations in support of services like vegetation, soil and water cover, inland waterways and coastal areas assessment, land use and change detection mapping, disaster relief support or climate change monitoring. S2 ensures the continuity for Landsat and Spot observations and improves the data availability. With a systematic global coverage and a 5 days temporal resolution, this mission raises a great interest due the augmented volume of medium resolution imagery it provides and its ability to measure the radiation reflected by the Earth surface in 13 different intervals of the electromagnetic spectrum.

S2 data is expected to strengthen the routine generation of products such as generic land-cover, land-use maps. They can contribute to the generation of geophysical variables like leaf coverage, leaf chlorophyll content and leaf water content.

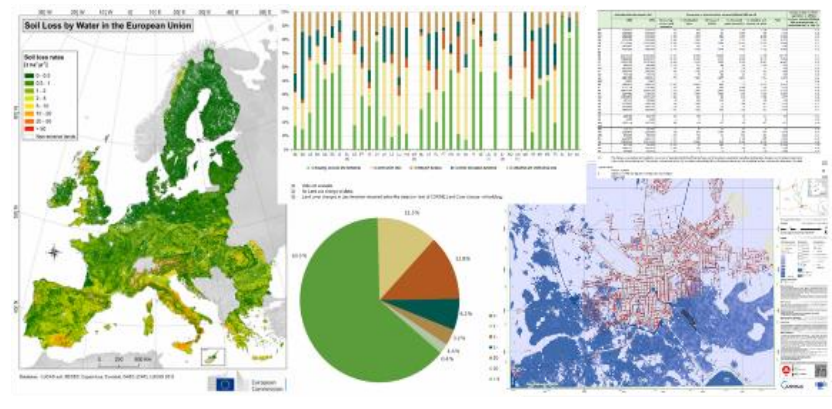

Figure 1. Example of EO derived products: regional / global thematic maps (vector or raster), charts and analytics.

In order to serve any type of application, the extracted information must be wrapped into value-added products integrating accurate land use, land cover, thematic maps and derived analytics on the scene content (Figure 1).

Given the huge quantity of data to be collected, human analysis is not convenient, especially when the information recorded is beyond the visible domain, and thus, beyond human ability to interpret. Computer based data analysis is highly required to extract similarity by processing and assist human understanding and semantic annotation. The core interest nowadays turns out to be an appropriate integration of multiple procedures that will ease the extraction of actionable intelligence and speed up the implementation of envisaged applications. With the goal to encourage communication between end-users, scientific communities and developers while promoting results and achievements, a community platform was created to increase Sentinel data exploitation and accelerate further scientific development. Named the Sentinel Application Platform (SNAP), it reunites dedicated Toolboxes in order to offer the most complex platform for all the Sentinel missions, including S2 [1].

A general approach considers that the content of the data must be firstly described by means of its main characteristics in order to extract objects and then modelled as feature vectors using various algorithms. The literature shows significant progress on theoretical methods and algorithms for the exploitation of multi-temporal data, but not many of them were developed or accustomed for the particularities of 
S2 data, despite their promising accuracy of results and they act in an isolated manner.

This paper proposed an exploratory search methodology for S2 data underpinning both visual and latent characteristics by means of data visualization and content representation. For optimized results, we present a detailed assessment of some of the most relevant state-of-the-art algorithms for features extraction and classification to determine which one could handle best the characteristics of S2 data. Exploratory visual analysis is considered to compute the best data representation given the content semantics. The approach is detailed in Section 2, while the evaluation report is presented in Section 3. We continue with experimental results on section 4 and conclude in section 5 .

\section{EXPLORATORY SEARCH METHODOLOGY FOR SENTINEL 2 DATA}

This paper introduces an unitary data mining framework to be applied towards the enhancement of relevant aspects that will facilitate transposing the Sentinel 2 data into actionable information. It was built to exploit both understandable, perceivable characteristics of the scene and numerical features hidden inside the data code. This entails for a two stages methodology searching for those particularities in S2 data that are relevant with respect to the semantic content of the analyzed scene (Figure 2). The proposed methodology requires that all bands of an S2 image to be brought to a common spatial resolution.

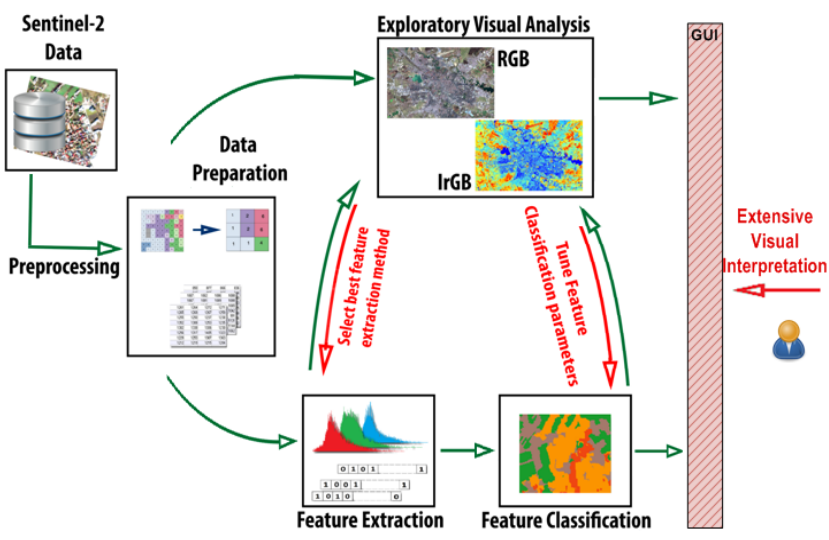

Figure 2. Exploratory search methodology for advanced S2 data analysis: combining visual characteristics (Exploratory Visual Analysis) with latent features (Feature extraction and classification) in view of extensive visual interpretation.

At the first stage, an exploratory visual analysis will highlight predominant data features for the scene content. The discrimination between ground structures is determined by their spectral signature. Hence advanced data visualization that will help the user to perceive certain aspects that are not always reflected in the visible part of the spectrum. Each of the semantic classes (e.g. water, forest, urban) distinguishable in the Sentinel 2 data has a specific spectral signature, which may not be focused on the visible spectral bands, so the "true color" image representation may hide important details. Data visualization includes 2 approaches: one dedicated to the discovery of the optimum 3 band combination able to highlight specific object categories, and the other focusing on merging all 13 bands into 3 through dimensionality reduction.

For the second stage, a data content representation will focus on the identification of relevant scene characteristics and further grouping by means of classification methods. A compact process interconnects feature extraction and feature classification in such a way to best describe the Sentinel 2 data content characteristics. Feature extraction is usually employed to extract and infer knowledge about patterns that are hidden inside the image, offering insights about the scene and advanced content description. Specific classes in the image share characteristics like coarseness, contrast, color distribution or directionality, which will make feature extraction methods sensitive to spectral, texture, and shape information. The informational content will be encapsulated into multi-dimensional feature vectors (mathematical representations of the image properties). Yet, feature vectors are a low level semantic representation. In order to reach actionable information, more compact structures must be identified, as a combination between proportions of different feature vectors. This is subject to a classification task, where a set of patterns are assigned into a group so that, according to some similarity metrics, the elements in the same cluster are more similar to each other than to those in other clusters. To this extent, similar extracted features are grouped together and receive a meaning and generate a classification map.

The two stages complement each other. While exploratory visual analysis is addressing visual properties of structures to stress features beyond visible, data content representation is focusing more on extracting numerical patterns which will lead to similarity by data processing and results not always corresponding to the user perception on the scene. Latent features are revealed, with no evident meaning what so ever. As such, an extensive visual interpretation is required, where, for each semantic class, a different data visualization can be computed to explain the classification result through all land cover, land use, geographical variables and land transformations.

\section{REVIEW ON CURRENT APPROACHES}

Abstractly, the proposed methodology for exploratory search throughout S2 data could accommodate a wide list of data visualization and content representation algorithms. Literature presents significant progress within these topics.

Feature extraction. In the EO domain, a lot of effort has been made to develop better texture, colour, and shape feature extraction techniques for both pixel and patchbased multispectral image analyses. Even though there are a lot of implementations, most of the texture analysis applications use techniques based on grey-level cooccurrence matrix [1], wavelet transforms [2] or Gabor 
filtering [3]. Colour features are very easy to compute in comparison with texture and shape features as they rely only on spectral information. They are widely employed in scene classification and content-based image retrieval applications. Colour histograms [4] are the most frequent in remote sensing image analysis. Local feature descriptors are another very important category of features. The most popular and widely used techniques are referred to scale and rotation invariant feature transform and local binary pattern [5].

Currently evolving texture analysis and local feature extraction (FE) techniques have led the way to the bag-ofwords (BoW) method. In the remote sensing community, this technique has been recently introduced for image annotation, object classification, target detection, and land use classification, and it has already proven its discrimination power in image classification [6]. In the BoW framework, there are several ways to generate the visual codebook, but kmeans-based approaches are preferred. An assessment of several patch-based approaches for FE is presented in [7]. The study in [8] tackles the problem of choosing the optimum number of number of classes that can be extracted and optimum patch size for Sentinel 2 data analysis.

Feature classification. Feature vectors provide lowlevel characteristics with very low capability in representing the semantic content, but they provide the key properties assisting further data modelling at a reduced computational burn. The next step is to determine similarities between those properties and group features up to a level where numeric patterns become meaningful. Similarity is identified via clustering procedures, where each feature vector is replaced by its label corresponding to one of the clusters. The prevailing algorithm in this category is k-means [9], which assigns each data point to the cluster whose centre is closer.

However, the semantic gap between the content description provided by low level features and the high level semantic content of the image can be confined by a series of user driven clustering techniques. One of most common is Relevance Feedback (RF). The RF is an iterative problem of supervised classification in two classes: relevant and irrelevant images for a certain query. The user's feedback is the key element in each iteration. Support Vector Machine (SVM) is one of the most popular approaches. The SVM has the advantage of a compact representation of the decision boundary and of learning by using few training examples [10]. A solution for reducing the annotation effort in RF and make it more efficient in the case of large archives is Active Learning (AL), a technique aiming to find the most informative images in the archive and to populate with them in the set of relevant images in the feedback. In [11], the computations in the AL loop are reduced by using a cascade of classifiers. Unknown semantic classes are learned by AL in [12] for auto-annotating of satellite image databases. The method idea is to incorporate in the learning process the nonannotated data which by definition contain unknown classes.

Exploratory data analysis focuses on the visualization of multidimensional remote sensing image by means of: feature selection (FS) algorithms or dimensionality reduction (DR) techniques. Both approaches decrease the dimensionality of the input data, underpinning though different composition of the feature subset.

FS algorithms identifies a data subset formed by the most relevant features of the input data. Minimum-redundancymaximum-relevance (mRMR) is the most used criterion for feature selection. This technique assumes that minimum redundancy leads to a set of features that best describe the data. mRMR applies mutual information to measure the relevance of EO data features due to its dependency on feature's changes according to elements' class [13].

These methods select only a reduced set of features, leading to the loss of information from the rest of the set. On the other hand, DR methods identify subset achieved by a linear transformation of the input data. A comparative review of the recent developed non-linear DR methods was presented in [14]. The performance of three DR techniques in the visualization field of the remote sensing image dataset was studied in [15]. Principal Component Analysis, Linear Discriminant Analysis and t-distributed Stochastic Neighbour Embedding algorithms ware used to delineate an optimal image content visualization method. The study concludes that DR algorithms highlight particular aspects of the analysed databases.

\section{EXPERIMENTAL RESULTS}

The proposed methodology aims at enabling a procedure dedicated to the properties of S2 data. As such, we performed an evaluation of the most promising feature extraction algorithms on the literature with respect to this purpose. Given the fact that each application comes with specific demands, we considered the Support Vector Machine as the reference feature classification method to build semantic relations between features based on the user's feedback. A common dataset was selected for the experiments and it includes a S2 image covering 30x30sq km over the city of Bucharest area and surroundings (Figure 4, a).

\begin{tabular}{|c|c|l|}
\hline Precision & Recall & \\
\hline $84.24 \%$ & $83.71 \%$ & BoW - Polar Coordinates \\
\hline $\mathbf{8 3 . 9 0} \%$ & $\mathbf{8 6 . 3 7 \%}$ & Gabor_superposition1 \\
\hline $\mathbf{8 2 . 6 2} \%$ & $\mathbf{8 0 . 6 5 \%}$ & BoW - Spectral indexes \\
\hline $82.14 \%$ & $81.37 \%$ & BoW - px Clusters \\
\hline $\mathbf{8 1 . 7 4 \%}$ & $\mathbf{8 5 . 7 3 \%}$ & Gabor_Hist \\
\hline $\mathbf{8 0 . 9 6 \%}$ & $\mathbf{8 0 . 3 3 \%}$ & WLD_Hist \\
\hline $\mathbf{8 0 . 7 7} \%$ & $\mathbf{7 8 . 8 8} \%$ & SCD polar coordinates \\
\hline $\mathbf{7 9 . 3 5 \%}$ & $\mathbf{8 2 . 5 0} \%$ & Spectral indexes \\
\hline $\mathbf{7 8 . 1 5} \%$ & $\mathbf{8 3 . 7 9 \%}$ & Polar coordinates \\
\hline $\mathbf{7 8 . 0 0} \%$ & $\mathbf{8 0 . 5 4 \%}$ & Gabor \\
\hline $68.95 \%$ & $62.72 \%$ & WLD \\
\hline $\mathbf{6 1 . 5 7} \%$ & $\mathbf{6 4 . 0 2} \%$ & BoW - MeanStDev \\
\hline $59.82 \%$ & $55.36 \%$ & BoW - px Values \\
\hline $58.81 \%$ & $57.94 \%$ & MPEG SCD bolarcoordinates \\
\hline
\end{tabular}

Figure 3. Average Precision / Recall values for the SVM classification results. 


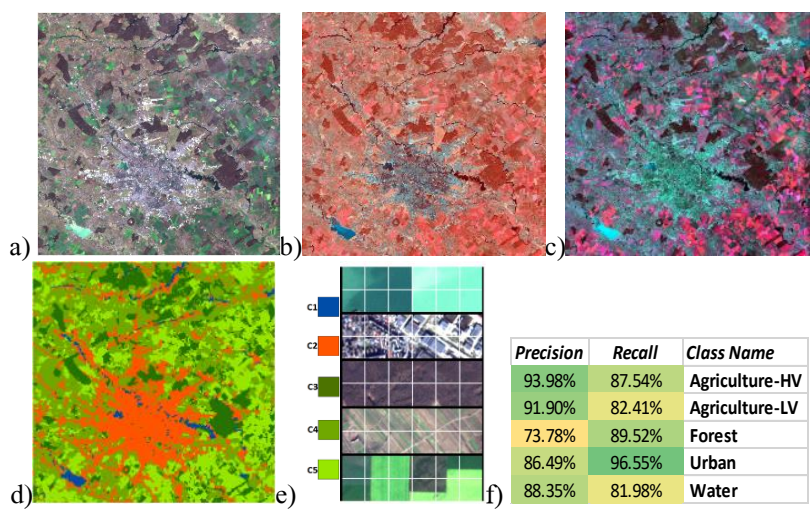

Figure 4. a) Bucharest area: Sentinel 2 data, RGB representation; b)c) Different visual representations computed to highlight waterspecific features; d) Scene classification; e) Illustration of relevant areas for each class label; f) Precision/Recall values for the classification result.

In addition, a manual annotation was performed to support the algorithms validation and evaluation. The reference map contains 5 semantic classes: water, urban, forest, low vegetation, high vegetation (Figure 4, e). Similar testing conditions were used for different feature extraction and classification methods, such that the selection was performed through precision/recall analysis. Figure 3 illustrates the average values for top $14 \mathrm{FE}$ algorithms.

Based on this assessment, a BoW approach applied to a polar coordinates transform [16] seems to be the best choice in case of S2 data analysis. In order to ensure full understanding of identified similarities and structure grouping inside the image, the user require full understanding of the scene, given its characteristics as measured throughout the 13 bands. For this reason, the classification results in Figure 4, d must be subject to an extensive visual interpretation in correlation with data visualization by means of FS (Figure 4, b) and DR methods (Figure 4, c). A prospect of both visual and latent characteristics will enable relevant exploratory S2 data search.

\section{CONCLUSIONS}

S2 data was designed to become the future of land cover mapping, enabling significant enhancement due to its spatial resolution, spectral coverage and revisit period of time. This paper tackles the need for tools to speed up data processing and deliver results to the users in a timely manner. The proposed methodology is meant to assist S2 data analysis and understanding by considering both visual and latent features. To this aim, a comparative assessment of state of the art algorithms for feature extractions outlines the expected performance in terms of precision and recall. Although it is not providing $100 \%$ accuracy, the polar coordinates used as a BoW approach were demonstrated to be the most suited for S2 image content representation. Parameter setup is expected to support any S2 data based application. Further perspectives are variate, as, for instance, transposing this proof of concept into an universal tool dedicated to multispectral data processing at a larger scale, for experienced users as well as for casual users.

\section{ACKNOWLEDGEMENT}

This work has been performed within the frame of the "Multispectral Data Analysis Toolbox for SNAP - ESA's SentiNel Application Platform" project, funded by ESA.

\section{REFERENCES}

[1] R. M. Haralick et al., "Textural features for image classification," IEEE Trans. Syst., Man, Cybern., vol. SMC-3, no. 6, pp. 610-621, Nov. 1973.

[2] P. Scheunders et al., "Wavelet-based texture analysis," Int. J. Comp. Sci. Inf. Manag., vol.1, pp.22-34,1998.

[3] Y. Hongyu, L. Bicheng, and C. Wen, "Remote sensing imagery retrieval based-on Gabor texture feature classification," in Proc. IEEE Signal Process., 2004, vol. 1, pp. 733-736.

[4] K. E. A. van de Sande et al., "Evaluating color descriptors for object and scene recognition," IEEE Trans. Pattern Anal. Mach. Intell., vol. 32, no. 9, pp. 1582-1596, Sep. 2009.

[5] T. Ojala et al., "Multiresolution gray-scale and rotation invariant texture classification with local binary patterns," IEEE Trans. Pattern Anal. Mach. Intell., vol. 24, no. 7, pp. 971-987, Jul. 2002.

[6] S. Cui, G. Schwarz, and M. Datcu, "Remote sensing image classification: No features, no clustering," IEEE J. Sel. Topics Appl. EO Rem Sens., vol. 8, no. 11, pp. 5158-5170, Nov. 2015.

[7] F.A. Georgescu, et al.,"Feature Extraction for Patch-based Classification of Multispectral Earth Observation Images", IEEE Geos and Rem Sensing Letters, vol. 13, pp 865-869, 2016

[8] F.A. Georgescu, M. Datcu, D. Raducanu,"Patch-Based Multispectral Image Classification Assessment for Sentinel-2 Data Analysis”, BiDS'16 International Conference, Tenerife, Spain, 2016 [9] S.P. Lloyd, "Least Squares Quantization in PCM", IEEE Trans. on Information Theory, vol. IT-28, no. 2, pp. 129-137, 1982.

[10] H.P.Graf, et al. "Parallel support vector machines: The cascade svm."Advances in neural information processing systems. 2004.

[11] Blanchart, Pierre, et al. "Pattern retrieval in large image databases using multiscale coarse-to-fine cascaded active learning." IEEE J of Sel Top. in Appl EO and Rem Sen, 7.4 (2014):1127-1141.

[12] Blanchart, Pierre, Mihai Datcu. "A semi-supervised algorithm for auto-annotation and unknown structures discovery in satellite image databases", IEEE Journal of Selected Topics in Applied Earth Observations and Remote Sensing, 3.4 (2010): 698-717.

[13] D. Bratasanu, et al., "Interactive Spectral Band Discovery for Exploratory Visual Analysis of Satellite Images," IEEE J. of Sel Top in Appl EO and Rem Sens, vol. 5, no. 1, pp. 207-224, Feb. 2012.

[14] L. Van der Maaten, et al, "Dimensionality reduction: A comparative review," Technical Report TiCC TR 2009-005.

[15] A. Griparis, D. Faur and M. Datcu, "Feature space dimensionality reduction for the optimization of visualization methods," IEEE Int. Geos and Rem Sen Symp, 2015, pp. 1120-1123.

[16] F.A. Georgescu, D. Raducanu, Mihai Datcu," New MPEG-7 Scalable Color Descriptor Based on Polar Coordinates for Multispectral Earth Observation Image Analysis", IEEE Geos and Rem Sensing Letters, vol. 14, no. 7, pp 987 - 991, 2017. 